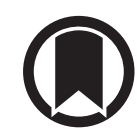

CrossMark

\title{
Incident opioid drug use and adverse respiratory outcomes among older adults with COPD
}

\author{
Nicholas T. Vozoris ${ }^{1,2,3}$, Xuesong Wang ${ }^{4}$, Hadas D. Fischer ${ }^{4}$, Chaim M. Bell ${ }^{3,4,5,6}$, \\ Denis E. O'Donnell 7 , Peter C. Austin ${ }^{4,5}$, Anne L. Stephenson ${ }^{1,2,3,5}$, \\ Sudeep S. Gill ${ }^{4,7}$ and Paula A. Rochon $3,4,5,8$
}

\begin{abstract}
Affiliations: 'Division of Respirology, Department of Medicine, St. Michael's Hospital, Toronto, ON, Canada. ${ }^{2}$ Keenan Research Centre in the Li Ka Shing Knowledge Institute, St Michael's Hospital, Toronto, ON, Canada. ${ }^{3}$ Dept of Medicine, University of Toronto, Toronto, ON, Canada. ${ }^{4}$ Institute for Clinical Evaluative Sciences, Toronto, ON, Canada. ${ }^{5}$ Institute of Health Policy, Management, and Evaluation, University of Toronto, Toronto, ON, Canada. 'Division of General Internal Medicine, Department of Medicine, Mount Sinai Hospital, Toronto, ON, Canada. ${ }^{7}$ Dept of Medicine, Queen's University, Kingston, ON, Canada. ${ }^{8}$ Women's College Research Institute, Women's College Hospital, Toronto, ON, Canada.
\end{abstract}

Correspondence: Nicholas Vozoris, Division of Respirology, Dept of Medicine, St. Michael's Hospital, 30 Bond Street, Toronto, ON, Canada, M5B 1W8. E-mail: nick.vozoris@utoronto.ca

ABSTRACT We evaluated risk of adverse respiratory outcomes associated with incident opioid use among older adults with chronic obstructive pulmonary diseases (COPD).

This was a retrospective population-based cohort study using a validated algorithm applied to health administrative data to identify adults aged 66 years and older with COPD. Inverse probability of treatment weighting using the propensity score was used to estimate hazard ratios comparing adverse respiratory outcomes within 30 days of incident opioid use compared to controls.

Incident opioid use was associated with significantly increased emergency room visits for COPD or pneumonia (HR 1.14, 95\% CI 1.00-1.29; p=0.04), COPD or pneumonia-related mortality (HR 2.16, 95\% CI 1.61-2.88; $\mathrm{p}<0.0001$ ) and all-cause mortality (HR 1.76, 95\% CI 1.57-1.98; $<<0.0001$ ), but significantly decreased outpatient exacerbations (HR 0.88 , 95\% CI 0.83-0.94; $\mathrm{p}=0.0002$ ). Use of more potent opioidonly agents was associated with significantly increased outpatient exacerbations, emergency room visits and hospitalisations for COPD or pneumonia, and COPD or pneumonia-related and all-cause mortality.

Incident opioid use, and in particular use of the generally more potent opioid-only agents, was associated with increased risk for adverse respiratory outcomes, including respiratory-related mortality, among older adults with COPD. Potential adverse respiratory outcomes should be considered when prescribing new opioids in this population.

@ERSpublications

New opioid drug use is associated with increased adverse respiratory outcomes and death among older adults with COPD http://ow.ly/4Jrg300Stbc

This article has supplementary material available from erj.ersjournals.com

Received: Nov 242015 | Accepted after revision: May 162016 | First published online: July 132016

Support statement: This research was funded by a grant from The Lung Association-Canadian Thoracic Society National Grant Review/Grant-In-Aid. This study was supported by the Institute for Clinical Evaluative Sciences (ICES), which is funded by an annual grant from the Ontario Ministry of Health and Long-Term Care (MOHLTC). The opinions, results and conclusions reported in this paper are those of the authors and are independent from the funding sources. No endorsement by ICES or the Ontario MOHLTC is intended or should be inferred. Parts of this material are based on data and information compiled and provided by Canadian Institute for Health Information (CIHI). However, the analyses, conclusions, opinions and statements expressed herein are those of the author, and not necessarily those of CIHI. Parts of this material are based on data and information provided by Cancer Care Ontario (CCO). The opinions, results, view, and conclusions reported in this paper are those of the authors and do not necessarily reflect those of CCO. No endorsement by CCO is intended or should be inferred. We thank Brogan Inc., Ottawa for use of their Drug Information Database. P.C. Austin was supported in part by a Career Investigator Award from the Heart and Stroke Foundation. Funding information for this article has been deposited with the Open Funder Registry.

Conflict of interest: Disclosures can be found alongside the online version of this article at erj.ersjournals.com

Copyright CERS 2016 


\section{Introduction}

New opioid use was found to be common among older adults with chronic obstructive pulmonary disease (COPD) in a recent population-based study [1]. Opioids may be prescribed to individuals with COPD for several reasons, including for chronic musculoskeletal pain, insomnia and refractory respiratory symptoms, all of which are common in COPD [2-7]. Opioids may have adverse respiratory effects on individuals with COPD through several mechanisms: respiratory depression [8-11]; reduced mucous clearance from cough suppression [12]; and immunosuppressive effects [13]. Several clinical trials have reported that systemic opioids are safe in individuals with advanced COPD [14-19], but the generalisability of these trial results is limited by several study design factors (e.g., small numbers of subjects enrolled [14-19], limited opioid dosing levels evaluated [14-16] and the exclusion from some analyses of subjects who perceived no benefit [17-19], experienced negative events [17-19] or died [18, 19]). Prevalent opioid use of $>30 \mathrm{mg}$ oral morphine equivalents per day compared with non-use among individuals with advanced COPD was found in one large cohort study to be associated with increased all-cause mortality, but this risk did not extend to opioid receipt of $\leqslant 30 \mathrm{mg}$ oral morphine equivalents per day [20]. Respiratory-specific outcomes were not examined in this trial [20]. Careful opioid use in the setting of advanced COPD is supported by several respiratory guidelines [21-24], but the 2014 Global Initiative for Chronic Obstructive Lung Disease (GOLD) guidelines concurrently advise that opioids have serious adverse effects and that few may derive benefits [24]. The purpose of this study was to evaluate the association of new opioid use with the risk of adverse respiratory outcomes among older adults with COPD.

\section{Methods}

Study design

This was a population-based retrospective cohort study. We used Ontario health administrative data (housed at the Institute for Clinical Evaluative Sciences (ICES) in Toronto, ON, Canada) from April 1, 2007 to April 31, 2012. The study start time of April 1 was based on ICES fiscal year data holdings. The province of Ontario has a culturally diverse population of about 13.5 million (about $40 \%$ of Canada's total population). The Research Ethics Board at Sunnybrook Health Sciences Centre (Toronto, ON, Canada) granted ethics approval.

\section{Data sources}

Multiple healthcare administrative databases were linked using unique encoded identifiers, including a previously validated database of Ontario adults with physician-diagnosed COPD. Online supplement 1 includes a brief description of the validation methods. For the current study, COPD diagnosis was based on a highly specific algorithm using three or more ambulatory claims for COPD within any 2-year period or one or more hospitalisations for COPD (specificity 95.4\% (95\% confidence intervals 92.6-97.4\%); sensitivity 57.5\% (95\% CI 47.9-66.8\%)) [25]. The Ontario Drug Benefit (ODB) database contains information on all publicly funded outpatient medication dispensings to Ontarians aged 65 years and older. Drug claim coding error in the ODB was low at $0.7 \%$ (95\% CI 0.5-0.9\%) [26]. Other healthcare administrative databases used in this study are described in online supplement 1.

\section{Study population}

To be included in the study, individuals had to satisfy the following three criteria during the period of April 1, 2007, and March 31, 2012: be an Ontario resident; have validated physician-diagnosed COPD; and be 66 years of age or older. Because information on incident medication use was not available for individuals younger than 66 years of age in the ODB database, these persons were not included in the study. Although individuals with COPD younger than 66 years old were not included, COPD largely affects adults over the age of 65 years [27]. Individuals receiving palliative care (based on physician service codes) in the year prior to the index date (defined below) were excluded, since goals of care and indications for opioid use may differ in this context. Community-dwelling older adults and those living in long-term care facilities were examined separately (see online Supplement 2 for the long-term care cohort analysis).

\section{Opioid drug exposure and index date definitions}

\section{Exposed group}

We included oral- and transdermal-formulation opioid drugs contained in the ODB database (table 1). Injectable and rectally administered opioids were not considered, because these would be unlikely to be used for COPD in outpatient care. We excluded partial agonist-partial antagonist opioid agents (i.e., suboxone) and combination opioid and glutamate receptor agonists (i.e., methadone), since these medications have distinct indications. Opioid drug users were defined by incident use of any opioid listed in table 1 between April 1, 2007, and March 31, 2012. As previously [1], incident use was defined as a drug dispensing claim with no receipt of any of the opioids listed in table 1 in the year prior to the incident date. Incident use was 
TABLE 1 Opioid drugs covered under the Ontario Drug Benefit programme

Oral tablet/capsule agents

\begin{tabular}{|c|c|c|c|c|}
\hline & & \\
\hline \multicolumn{2}{|c|}{ Opioid-only agonists } & \multirow{2}{*}{$\begin{array}{l}\text { Opioid/non-opioid } \\
\text { combination agents }\end{array}$} & & \\
\hline Shorter-acting agents & Longer-acting agents & & & \\
\hline Codeine phosphate & Hydromorphone $\mathrm{HCl}$ ER/SR & Acetaminophen-codeine & Codeine phosphate & \\
\hline Hydromorphone $\mathrm{HCl}$ & Levorphanol & Acetylsalicylic acid-codeine-caffeine & Morphine $\mathrm{HCl}$ & \\
\hline Oxycodone $\mathrm{HCl}$ & $\begin{array}{l}\text { Propoxyphene } \mathrm{HCl} \text {; } \\
\quad \text { dextro-propoxyphene } \mathrm{HCl}\end{array}$ & Oxycodone $\mathrm{HCl}$-acetylsalicylic acid & & \\
\hline
\end{tabular}

Rectal and intravenous opioid formulations were not considered. ER: extended release; HCL: hydrochloride; SR: slow release. ${ }^{\# A l l}$ oral liquid opioid formulations are shorter acting. ๆ: transdermal fentanyl is a longer-acting opioid agent.

only counted once per individual. If an individual met criteria for incident use more than once, then only the first dispensation was considered. Individuals with incident opioid use at least once between April 1, 2007, and March 31, 2012, were excluded from entering the unexposed comparison group at any time. We studied incident opioid use, rather than prevalent use, because our purpose was to examine acute-onset adverse respiratory outcomes and because incident use is less likely to be associated with "healthy user" bias. The index date for the exposed subjects was the date the incident opioid was dispensed.

\section{Control group}

Controls were not dispensed any opioids listed in table 1 between April 1, 2007, and March 31, 2012. Using a similar approach as used previously [28, 29], cohort entry for controls was defined as the most recent of any incident non-opioid medication claim on or before a date chosen randomly from the accrual period. The most recent incident non-opioid medication claim served as the index date. Incident use was defined as no receipt of a medication within the same ODB subclass as the index non-opioid drug in the year prior to index. If the most recent claim took place more than 6 months before that date, or if it took place before the start of the 2007-2012 period, then the individual was not included.

\section{Propensity score weighting}

Given known differences in patient characteristics between opioid users and controls [1], inverse probability of treatment weighting using the propensity score $[30,31]$ was employed to create weighted samples of exposed and control individuals where measured baseline covariates were balanced between the two groups. We estimated a propensity score for new opioid receipt by developing a logistic regression model with 33 covariates describing patient demographic and health characteristics that are known to be associated with opioid receipt in older adults with COPD [1] and the outcomes of interest (table 2). Indicators of COPD severity, health care use, and comorbidities were included in the propensity score. See online supplement 1 for more details on our propensity score weighting methods.

\section{Study outcomes}

We examined the following adverse respiratory outcomes occurring within 30 days after the index date: outpatient respiratory exacerbations (defined similar to others [32,33], as receipt of an oral corticosteroid or respiratory antibiotic within \pm 7 days of a physician clinic/office visit for COPD or pneumonia and with the corticosteroid or antibiotic prescription having a supply date of 5-21 days); emergency room visits for COPD or pneumonia; hospitalisations for COPD or pneumonia; intensive care unit (ICU) admission during a hospitalisation for COPD or pneumonia; COPD or pneumonia-related mortality; and all-cause mortality. The following International Classification of Diseases (ICD)-10 codes were used to identify emergency room visits and hospitalisations for which the diagnosis was COPD or pneumonia: J41, J42, J43 and J44 for COPD; J09-18, J20-22 and J40 for pneumonia. COPD- or pneumonia-related mortality was identified if cause of death was coded with one of the following ICD-9 codes: 491, 492 and 496 for COPD; 4641, 4642, 4660, 4661, 480-87 and 490 for pneumonia. A 30-day follow-up period was selected since we expected possible opioid-related adverse events to occur soon after drug initiation. 
TABLE 2 Baseline demographic and health characteristics of the community-dwelling cohort, before and after propensity score weighting

Baseline characteristics

Before propensity score weighting

After propensity score weighting

\begin{tabular}{|c|c|c|c|c|c|c|}
\hline & & & & & & \\
\hline & $\begin{array}{c}\text { New opioid } \\
\text { users }\end{array}$ & Controls & $\begin{array}{l}\text { Standardised } \\
\text { difference }\end{array}$ & $\begin{array}{c}\text { New opioid } \\
\text { users }\end{array}$ & Controls & $\begin{array}{l}\text { Standardised } \\
\text { difference }\end{array}$ \\
\hline Subjects & 89327 & 41652 & & 89224 & 41930 & \\
\hline $\begin{array}{l}\text { Low income based on ODB flag within } 1 \text { year } \\
\text { prior to the index date }\end{array}$ & 24.2 & 21.8 & 0.06 & 23.4 & 23.4 & 0.00 \\
\hline \multicolumn{7}{|l|}{ Income quintile } \\
\hline 3 & 19.5 & 20.0 & 0.01 & 19.7 & 19.7 & 0.00 \\
\hline 4 & 18.0 & 18.4 & 0.01 & 18.1 & 17.9 & 0.01 \\
\hline 5 (highest) & 16.5 & 17.0 & 0.01 & 16.7 & 16.6 & 0.00 \\
\hline Missing data & 0.3 & 0.3 & 0.00 & 0.3 & 0.3 & 0.00 \\
\hline Rural setting & 16.7 & 17.4 & 0.02 & 16.9 & 16.9 & 0.00 \\
\hline \multicolumn{7}{|l|}{ COPD exacerbation frequency in past year } \\
\hline$<2$ years & 22.5 & 38.9 & 0.37 & 27.7 & 28.0 & 0.01 \\
\hline $2-5$ years & 19.6 & 15.9 & 0.10 & 18.4 & 18.5 & 0.00 \\
\hline$>5$ years & 57.9 & 45.2 & 0.26 & 53.9 & 53.5 & 0.01 \\
\hline \multicolumn{7}{|l|}{ Medications in past 180 days } \\
\hline Short/long-acting $\beta$ agonists & 36.0 & 34.6 & 0.03 & 35.6 & 36.1 & 0.01 \\
\hline Short/long-acting anticholinergics & 39.2 & 37.9 & 0.03 & 39.0 & 39.5 & 0.01 \\
\hline Inhaled corticosteroids & 14.0 & 13.6 & 0.01 & 13.9 & 13.9 & 0.00 \\
\hline $\begin{array}{l}\text { Combination inhaled corticosteroid-long } \\
\text { acting beta agonist inhalers }\end{array}$ & 33.2 & 30.1 & 0.07 & 32.3 & 32.7 & 0.01 \\
\hline Oral corticosteroids & 13.8 & 11.8 & 0.06 & 13.2 & 13.5 & 0.01 \\
\hline Theophylline & 2.4 & 1.9 & 0.03 & 2.3 & 2.3 & 0.00 \\
\hline Respiratory antibiotics & 44.9 & 40.7 & 0.09 & 43.6 & 44.2 & 0.01 \\
\hline \multicolumn{7}{|l|}{ Charlson score } \\
\hline 0 & 29.8 & 29.6 & 0.00 & 29.6 & 28.8 & 0.02 \\
\hline 1 & 18.8 & 17.6 & 0.03 & 18.5 & 18.4 & 0.00 \\
\hline 2 & 13.7 & 12.7 & 0.03 & 13.5 & 13.7 & 0.01 \\
\hline$\geqslant 3$ & 22.0 & 20.1 & 0.05 & 21.6 & 22.6 & 0.03 \\
\hline Missing data & 15.7 & 19.9 & 0.11 & 16.9 & 16.4 & 0.01 \\
\hline Ischaemic heart disease ${ }^{\pi}$ & 47.5 & 42.9 & 0.09 & 46.1 & 46.5 & 0.01 \\
\hline Congestive heart failure ${ }^{\pi}$ & 26.8 & 27.2 & 0.01 & 27.0 & 27.5 & 0.01 \\
\hline Any malignancy $\pi$ & 13.0 & 7.7 & 0.17 & 11.4 & 12.1 & 0.02 \\
\hline Musculoskeletal/connective tissue disease ${ }^{\pi}$ & 86.9 & 78.2 & 0.24 & 84.2 & 84.4 & 0.01 \\
\hline Osteoporosis" & 16.0 & 15.1 & 0.02 & 15.8 & 15.7 & 0.00 \\
\hline Psychotic psychiatric disease ${ }^{\pi}$ & 5.5 & 5.6 & 0.01 & 5.6 & 5.7 & 0.01 \\
\hline Non-psychotic psychiatric disease $e^{\pi}$ & 50.5 & 47.4 & 0.06 & 49.6 & 49.8 & 0.00 \\
\hline Sleep disorder ${ }^{7}$ & 55.2 & 53.0 & 0.04 & 54.6 & 54.9 & 0.01 \\
\hline Dementia" & 11.2 & 13.8 & 0.08 & 12.0 & 12.1 & 0.00 \\
\hline Cohort entry in flu season & 38.1 & 38.7 & 0.01 & 38.2 & 38.1 & 0.00 \\
\hline
\end{tabular}

Data are presented as mean \pm SD or \%, unless otherwise stated. ODB: Ontario Drug Benefit; COPD: chronic obstructive pulmonary disease; ICU: intensive care unit. " : standardised differences of $>0.10$ are thought to indicate potentially meaningful differences. ": presence of all comorbidities was based on 5-year look-back from the index date. 


\section{Sensitivity analyses}

Several sensitivity analyses were undertaken. First, we examined for adverse outcomes by opioid type received (i.e., opioid-only agents versus combination opioid/non-opioid agents (table 1)). This analysis was undertaken in order to separately examine outcomes among recipients of opioid-only agents, which generally contain more potent opioids (like hydromorphone, fentanyl and levorphanol) unlike combination agents (most of which contain less-potent codeine).

Second, we examined for adverse outcomes by drug half-life among users of opioid-only agents (i.e., shorter-acting versus longer-acting agents (table 1)). This analysis was undertaken to separately examine outcomes in the subgroup of individuals receiving longer-acting opioids.

Third, among users of opioid-only agents, we examined for adverse outcomes by drug dose. Two drug dose levels were considered: $\leqslant 30 \mathrm{mg}$ of morphine equivalents per day and $>30 \mathrm{mg}$ morphine equivalents per day. These two drug dose levels have been previously used by others to denote lower and higher dosed opioids, respectively [20]. Online supplement 1 contains information on how different opioid drug doses were converted into $\mathrm{mg}$ of morphine equivalents per day. The drug dose analysis was undertaken separately for shorter-acting versus longer-acting agents.

Fourth, our analysis was stratified by COPD exacerbation history in the year prior to the index date (i.e., 0 exacerbations versus 1 or more outpatient exacerbations with no exacerbations requiring presentation to hospital versus 1 or more exacerbations requiring presentation to hospital). Outcomes in the subgroup of individuals with least severe disease (i.e., 0 exacerbations in the year prior to index) were examined in order to minimise possible effects of confounding by indication $[34,35]$. The propensity score was re-estimated for each of our sensitivity analyses. See online supplement 1 for other sensitivity analyses.

\section{Statistical analysis}

Descriptive statistics and standardised differences were calculated for the exposed and control groups on all baseline covariates before and after propensity score weighting [31]. A hazard ratio with associated 95\% confidence interval for each outcome was estimated for the propensity score weighted samples using Cox proportional hazard regression model with a robust variance estimator [36]. The control group was used as the reference in all analyses. All statistical analyses were conducted using SAS Enterprise Guide 6.1 (SAS Institute Inc., Cary, NC, USA). Two-sided tests of significance at the $\mathrm{p}<0.05$ level were used.

\section{Results}

\section{Overall cohort results}

A total of 130979 community-dwelling individuals with COPD who were 66 years and older were identified between April 1, 2007, and March 31, 2012, 68.2\% of whom received a new opioid (table 2). Baseline demographic and health characteristics of new opioid users and controls are described in table 2. Baseline characteristics were well-balanced between new users and controls following propensity score weighting, with standardised differences below $10 \%$ for all variables.

Compared with controls, new opioid users in the community were associated with significantly increased risk of emergency room visits for COPD or pneumonia (HR 1.14, 95\% CI 1.00-1.29; p=0.04) (table 3). Outpatient exacerbation risk was decreased among opioid users (HR 0.88, 95\% CI 0.83-0.94) and there were no significant associations observed with hospitalisations, or ICU admissions during hospitalisations, for COPD or pneumonia. Opioid use was also associated with significantly increased risk for COPD or pneumonia-related mortality (HR 2.16, 95\% CI 1.61-2.88) and all-cause mortality (HR 1.76, 95\% CI 1.57-1.98).

\section{Sensitivity analysis results}

In the subgroup analysis of users of opioid-only agents relative to controls, there were significantly increased associations with outpatient respiratory exacerbations (HR 1.27, 95\% CI 1.14-1.41), ER visits (HR 1.64, 95\% CI 1.35-1.98) and hospitalisations (HR 1.54, 95\% CI 1.31-1.81) for COPD or pneumonia, COPD or pneumonia-related mortality (HR 4.76, 95\% CI 3.40-6.66) and all-cause mortality (HR 4.01, 95\% CI 3.53-4.56) (table 4). There was no association between new opioid use and ICU admissions during hospitalisations for COPD or pneumonia. Among users of combination opioid/non-opioid agents versus controls, the association with outpatient exacerbations was decreased (HR 0.83, 95\% CI 0.77-0.88), but risks of COPD or pneumonia-related mortality (HR 1.58, 95\% CI 1.16-2.14) and all-cause mortality (HR $1.31,95 \%$ CI 1.16-1.48) were elevated.

Compared with controls, new use of both shorter-acting and longer-acting opioid-only agents was associated with increased risk for hospitalisations for COPD or pneumonia (shorter-acting agents: HR 1.50, 95\% CI 1.26-1.79; longer-acting agents: HR 1.86, 95\% CI 1.23-2.81), as well as COPD or pneumonia-related mortality (shorter-acting agents: HR 4.78, 95\% CI 3.36-6.79; longer-acting agents: HR 5.42, 95\% CI 2.53-11.62) and 
TABLE 3 Hazard ratios and confidence intervals for adverse respiratory outcomes among propensity score weighted community-dwelling cohort

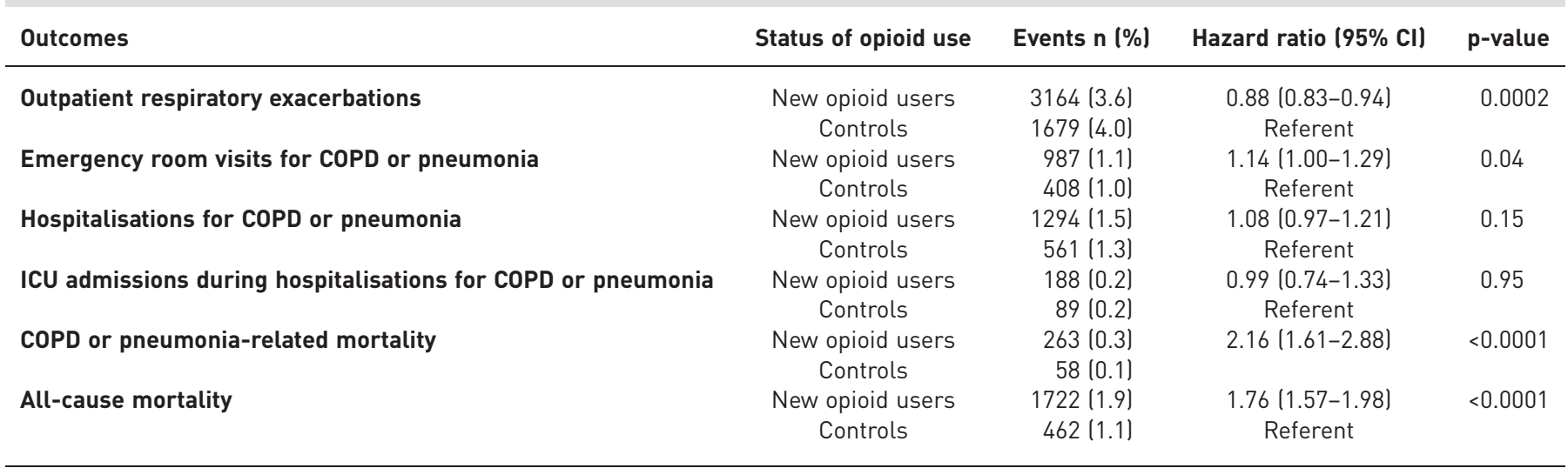

COPD: chronic obstructive pulmonary disease; ICU: intensive care unit.

all-cause mortality (shorter-acting agents: HR 3.80, 95\% CI 3.31-4.36; longer-acting agents: HR 5.62, 95\% CI 4.46-7.10) (table 5). Shorter-acting agents were also associated with increased risk for outpatient exacerbations (HR 1.37, 95\% CI 1.23-1.53) and ER visits for COPD or pneumonia (HR 1.70, 95\% CI 1.38-2.09), but not longer-acting agents. No significant relationships were observed with ICU admissions during hospitalisations for COPD or pneumonia.

Compared with controls, users of longer-acting opioid-only agents of $\leqslant 30 \mathrm{mg}$ of morphine equivalents per day were associated with significantly increased risk of hospitalisations for COPD or pneumonia (HR 1.93, 95\% CI 1.05-3.54), as well increased COPD or pneumonia-related mortality (HR 6.46, 95\% CI 2.94-14.21) and all-cause mortality (HR 6.89, 95\% CI 5.03-9.44) (table 6). Among users of longer-acting of opioid-only agents of $>30 \mathrm{mg}$ of morphine equivalents per day, there were associations with decreased risk of outpatient exacerbations (HR 0.65, 95\% CI 0.44-0.98), but greater risk for hospitalisations for COPD or pneumonia (HR 1.82, 95\% CI 1.04-3.17), COPD or pneumonia-related mortality (HR 4.72, 95\% CI 1.2418.05) and all-cause mortality ( $\mathrm{HR} 4.86$, 95\% CI 3.47-6.79). Compared with controls, users of shorter-acting opioid-only agents of $\leqslant 30 \mathrm{mg}$ of morphine equivalents per day were associated with significantly increased risk for hospitalisations for COPD or pneumonia (HR 1.73, 95\% CI 1.26-2.36), COPD or pneumonia-related mortality (HR 7.55, 95\% CI 4.84-11.79) and all-cause mortality (HR 5.19, 95\% CI 4.26-6.31). Users of shorter-acting opioid-only agents of $>30 \mathrm{mg}$ of morphine equivalents per day were associated with greater risk for outpatient exacerbations (HR 1.45, 95\% CI 1.28-1.64), emergency room visits (HR 1.87, 95\% CI 1.47-2.39) and hospitalisations (HR 1.47, 95\% CI 1.19-1.81) for COPD or pneumonia, COPD or pneumonia-related mortality (HR 3.26, 95\% CI 2.04-5.20) and all-cause mortality (HR 3.21, 95\% CI 2.72-3.79).

Among individuals with 0 exacerbations in the year prior to the index date, there were fewer outpatient exacerbations associated with new users versus controls (HR 0.85, 95\% CI 0.76-0.95) (table 7). Compared with controls, the association with emergency room visits for COPD or pneumonia was decreased among new users with $\geqslant 1$ outpatient exacerbations in the year prior to index (HR 0.73, 95\% CI 0.54-0.98), but increased among new users with $\geqslant 1$ exacerbations requiring presentation to hospital (HR 1.25, 95\% CI $1.05-1.48)$. COPD or pneumonia-related mortality risk was significantly greater among new users with $\geqslant 1$ outpatient exacerbations in the year prior to index (HR 3.15, 95\% CI 1.09-9.14) and among new users with $\geqslant 1$ exacerbations requiring presentation to hospital (HR 2.55, 95\% CI 1.71-3.80). New users were associated with significantly higher risk for all-cause mortality versus controls across all COPD exacerbation frequency subgroups ( 0 exacerbations in the year prior to index: HR 1.67, 95\% CI 1.39-2.02; $\geqslant 1$ outpatient exacerbation in the year prior to index: HR $1.79,95 \%$ CI $1.35-2.39$; and $\geqslant 1$ exacerbation requiring presentation to hospital: HR $1.89,95 \%$ CI $1.59-2.24)$.

\section{Discussion}

Our population-based cohort of 130979 community-dwelling older adults with COPD showed that new opioid use and, in particular, use of the generally more potent opioid-only agents, was associated with increased adverse respiratory outcomes and mortality. While a previous study had found high-dose opioid use to be associated with increased all-cause mortality [20], this is the first study to our knowledge to report on a link between opioid use and respiratory-related morbidity and mortality, regardless of opioid dose. 
TABLE 4 Hazard ratios and confidence intervals for adverse respiratory outcomes among propensity score weighted community-dwelling cohort, distinguishing by opioid drug type

\begin{tabular}{|c|c|c|c|c|c|c|c|c|c|c|c|c|c|c|c|c|c|c|c|}
\hline \multirow[t]{2}{*}{ Opioid drug type } & \multirow[t]{2}{*}{$\begin{array}{l}\text { Opioid use } \\
\text { status }\end{array}$} & \multicolumn{3}{|c|}{ Outpatient respiratory exacerbations } & \multicolumn{3}{|c|}{$\begin{array}{l}\text { Emergency room visits for } \\
\text { COPD or pneumonia }\end{array}$} & \multicolumn{3}{|c|}{$\begin{array}{l}\text { Hospitalisations for } \\
\text { COPD or pneumonia }\end{array}$} & \multicolumn{3}{|c|}{$\begin{array}{l}\text { ICU admissions during } \\
\text { hospitalisations for } \\
\text { COPD or pneumonia }\end{array}$} & \multicolumn{3}{|c|}{$\begin{array}{l}\text { COPD or pneumonia-related } \\
\text { mortality }\end{array}$} & \multicolumn{3}{|c|}{ All-cause mortality } \\
\hline & & $\begin{array}{l}\text { Events n } \\
\qquad \%]\end{array}$ & $\begin{array}{l}\text { Hazard ratio } \\
(95 \% \mathrm{CI})\end{array}$ & p-value & $\begin{array}{c}\text { Events n } \\
\quad \%(\%)\end{array}$ & $\begin{array}{l}\text { Hazard ratio } \\
(95 \% \mathrm{Cl})\end{array}$ & p-value & $\begin{array}{l}\text { Events n } \\
{[\%]}\end{array}$ & $\begin{array}{l}\text { Hazard ratio } \\
(95 \% \mathrm{Cl})\end{array}$ & $p$-value & $\begin{array}{l}\text { Events n } \\
\quad(\%)\end{array}$ & $\begin{array}{l}\text { Hazard ratio } \\
(95 \% \mathrm{Cl})\end{array}$ & p-value & $\begin{array}{l}\text { Events } n \\
\quad(\%)\end{array}$ & $\begin{array}{l}\text { Hazard ratio } \\
(95 \% \mathrm{Cl})\end{array}$ & p-value & $\begin{array}{l}\text { Events n } \\
\quad(\%)\end{array}$ & $\begin{array}{l}\text { Hazard ratio } \\
(95 \% \mathrm{Cl})\end{array}$ & $p$-value \\
\hline $\begin{array}{l}\text { Opioid-only } \\
\text { formulation }\end{array}$ & $\begin{array}{l}\text { New users } \\
\text { Controls }\end{array}$ & $\begin{array}{l}662(5.3) \\
1752(4.2)\end{array}$ & $\begin{array}{c}1.27(1.14-1.41) \\
\text { Referent }\end{array}$ & $<0.0001$ & $\begin{array}{l}214(1.7) \\
438(1.0)\end{array}$ & $\begin{array}{l}1.64(1.35-1.98) \\
\text { Referent }\end{array}$ & $<0.0001$ & $\begin{array}{l}282(2.3) \\
614(1.5)\end{array}$ & $\begin{array}{l}1.54 \text { (1..31-1.81) } \\
\text { Referent }\end{array}$ & $<0.0001$ & $\begin{array}{l}37(0.3) \\
97(0.2)\end{array}$ & $\begin{array}{l}1.27(0.82-1.96) \\
\text { Referent }\end{array}$ & 0.28 & $\begin{array}{l}97(0.8) \\
70(0.2)\end{array}$ & $\begin{array}{l}4.76(3.40-6.66) \\
\text { Referent }\end{array}$ & $<0.0001$ & $\begin{array}{l}614(4.9) \\
523(1.3)\end{array}$ & $\begin{array}{l}\text { 4.01 (3.53-4.56) } \\
\text { Referent }\end{array}$ & $<0.0001$ \\
\hline $\begin{array}{l}\text { Combination } \\
\text { opioid// } \\
\text { non-opioid } \\
\text { formulation }\end{array}$ & $\begin{array}{l}\text { New users } \\
\text { Controls }\end{array}$ & $\begin{array}{l}2527(3.3) \\
1662(4.0)\end{array}$ & $\begin{array}{l}0.83(0.77-0.88) \\
\text { Referent }\end{array}$ & $<0.0001$ & $\begin{array}{l}786(1.0) \\
399(1.0)\end{array}$ & $\begin{array}{l}1.08(0.95-1.23) \\
\text { Referent }\end{array}$ & 0.26 & $\begin{array}{l}1010(1.3) \\
544(1.3)\end{array}$ & $\begin{array}{l}1.01(0.91-1.14) \\
\text { Referent }\end{array}$ & 0.80 & $\begin{array}{l}154(0.2) \\
86(0.2)\end{array}$ & $\begin{array}{l}0.98 \text { (0.73-1.32) } \\
\text { Referent }\end{array}$ & 0.90 & $\begin{array}{l}163(0.2) \\
57(0.1)\end{array}$ & $\begin{array}{l}1.58 \text { (1.16-2.14) } \\
\text { Referent }\end{array}$ & 0.003 & $\begin{array}{l}1070(1.4) \\
449(1.1)\end{array}$ & $\begin{array}{l}1.31(1.16-1.48) \\
\text { Referent }\end{array}$ & $<0.0001$ \\
\hline
\end{tabular}

COPD: chronic obstructive pulmonary disease; ICU: intensive care unit.

TABLE 5 Hazard ratios and confidence intervals for adverse respiratory outcomes among propensity score weighted community-dwelling cohort, distinguishing by opioid half-life drug type

\begin{tabular}{|c|c|c|c|c|c|c|c|c|c|c|c|c|c|c|c|c|c|c|c|}
\hline \multirow[t]{2}{*}{$\begin{array}{l}\text { Opioid drug } \\
\text { half-life type }\end{array}$} & \multirow[t]{2}{*}{$\begin{array}{l}\text { Opioid } \\
\text { use status }\end{array}$} & \multicolumn{3}{|c|}{ Outpatient respiratory exacerbations } & \multicolumn{3}{|c|}{$\begin{array}{l}\text { Emergency room visits for } \\
\text { COPD or pneumonia }\end{array}$} & \multicolumn{3}{|c|}{$\begin{array}{l}\text { Hospitalisations for } \\
\text { COPD or pneumonia }\end{array}$} & \multicolumn{3}{|c|}{$\begin{array}{l}\text { ICU admissions during hospitalisations } \\
\text { for COPD or pneumonia }\end{array}$} & \multicolumn{3}{|c|}{ COPD or pneumonia-related mortality } & \multicolumn{3}{|c|}{ All-cause mortality } \\
\hline & & $\begin{array}{l}\text { Events n } \\
(\%)\end{array}$ & $\begin{array}{l}\text { Hazard ratio } \\
(95 \% \mathrm{Cll})\end{array}$ & p-value & Events $\mathrm{n}(\%)$ & $\begin{array}{l}\text { Hazard ratio } \\
(95 \% \mathrm{Cl})\end{array}$ & p-value & $\begin{array}{c}\text { Events n } \\
(\%)\end{array}$ & $\begin{array}{l}\text { Hazard ratio } \\
\text { (95\% Cl) }\end{array}$ & p-value & $\begin{array}{c}\text { Events } n \\
(\%)\end{array}$ & $\begin{array}{l}\text { Hazard ratio } \\
\text { (95\% Cl) }\end{array}$ & p-value & Events $n(\%)$ & $\begin{array}{l}\text { Hazard ratio } \\
(95 \% \mathrm{Cl})\end{array}$ & p-value & $\begin{array}{l}\text { Events n } \\
\quad(\%)\end{array}$ & $\begin{array}{l}\text { Hazard ratio } \\
(95 \% \mathrm{Cl})\end{array}$ & p-value \\
\hline $\begin{array}{l}\text { Shorter-acting } \\
\text { opioid-only } \\
\text { formulation }\end{array}$ & $\begin{array}{l}\text { New users } \\
\text { Controls }\end{array}$ & $\begin{array}{l}585(5.7) \\
1755(4.2)\end{array}$ & $\begin{array}{l}1.37(1.23-1.53) \\
\text { Referent }\end{array}$ & $<0.0001$ & $\begin{array}{l}181(1.8) \\
437(1.0)\end{array}$ & $\begin{array}{l}1.70(1.38-2.09) \\
\text { Referent }\end{array}$ & $<0.0001$ & $\begin{array}{l}224(2.2) \\
611(1.5)\end{array}$ & $\begin{array}{l}1.50(1.26-1.79) \\
\text { Referent }\end{array}$ & $<0.0001$ & $\begin{array}{l}30(0.3) \\
97(0.2)\end{array}$ & $\begin{array}{l}1.28(0.80-2.05) \\
\text { Referent }\end{array}$ & 0.30 & $\begin{array}{l}80(0.8) \\
70(0.2)\end{array}$ & $\begin{array}{l}4.78(3.36-6.79) \\
\text { Referent }\end{array}$ & $<0.0001$ & $\begin{array}{l}474(4.6) \\
520(1.2)\end{array}$ & $\begin{array}{l}3.80(3.31-4.36) \\
\text { Referent }\end{array}$ & $<0.0001$ \\
\hline $\begin{array}{l}\text { Longer-acting } \\
\text { opioid-only } \\
\text { formulation }\end{array}$ & $\begin{array}{c}\text { New users } \\
\text { Controls }\end{array}$ & $\begin{array}{c}67(3.0) \\
1722(4.1)\end{array}$ & $\begin{array}{l}0.72(0.52-0.98) \\
\text { Referent }\end{array}$ & 0.04 & $\begin{array}{l}31(1.4) \\
422(1.0)\end{array}$ & $\begin{array}{l}1.38(0.87-2.17) \\
\text { Referent }\end{array}$ & 0.17 & $\begin{array}{l}59(2.6) \\
589(1.4)\end{array}$ & $\begin{array}{l}1.86(1.23-2.81) \\
\text { Referent }\end{array}$ & 0.004 & $\begin{array}{c}<6^{\#} \\
92(0.2)\end{array}$ & $\begin{array}{l}1.07(0.42-2.77) \\
\text { Referent }\end{array}$ & 0.88 & $\begin{array}{l}20(0.9) \\
70(0.2)\end{array}$ & $\begin{array}{l}5.42(2.53-11.62) \\
\text { Referent }\end{array}$ & $<0.0001$ & $\begin{array}{l}149(6.6) \\
510(1.2)\end{array}$ & $\begin{array}{l}5.62(4.46-7.10) \\
\text { Referent }\end{array}$ & $<0.0001$ \\
\hline
\end{tabular}

COPD: chronic obstructive pulmonary disease; ICU: intensive care unit. \#: percentages are not presented, according to Institute of Clinical Evaluative Sciences reporting rules, because of small cell size. 
TABLE 6 Hazard ratios and confidence intervals for adverse respiratory outcomes among propensity score weighted community-dwelling cohort, distinguishing by opioid drug dose

\begin{tabular}{|c|c|c|c|c|c|c|c|c|c|c|c|c|c|c|c|c|c|c|c|}
\hline \multirow[t]{2}{*}{ Opioid drug dose category } & \multirow[t]{2}{*}{$\begin{array}{l}\text { Opioid use } \\
\text { status }\end{array}$} & \multicolumn{3}{|c|}{ Outpatient respiratory exacerbations } & \multicolumn{3}{|c|}{$\begin{array}{l}\text { Emergency room visits } \\
\text { for COPD or pneumonia }\end{array}$} & \multicolumn{3}{|c|}{ Hospitalisations for COPD or pneumonia } & \multicolumn{3}{|c|}{$\begin{array}{l}\text { ICU admissions during hospitalisations } \\
\text { for COPD or pneumonia }\end{array}$} & \multicolumn{3}{|c|}{ COPD or pneumonia-related mortality } & \multicolumn{3}{|c|}{ All-cause mortality } \\
\hline & & $\begin{array}{l}\text { Events n } \\
\quad(\%)\end{array}$ & $\begin{array}{l}\text { Hazard ratio } \\
(95 \% \mathrm{CII})\end{array}$ & p-value & $\begin{array}{l}\text { Events n } \\
\qquad \%)\end{array}$ & $\begin{array}{l}\text { Hazard ratio } \\
(95 \% \mathrm{Cl})\end{array}$ & $\mathrm{p}$-value & $\begin{array}{l}\text { Events n } \\
\quad(\%)\end{array}$ & $\begin{array}{l}\text { Hazard ratio } \\
(95 \% \mathrm{CI})\end{array}$ & p-value & $\begin{array}{l}\text { Events n } \\
\quad(\%)\end{array}$ & $\begin{array}{l}\text { Hazard ratio } \\
(95 \% \mathrm{Cl})\end{array}$ & p-value & $\begin{array}{l}\text { Events } \mathrm{n} \\
(\%)\end{array}$ & $\begin{array}{l}\text { Hazard ratio } \\
(95 \% \mathrm{Cl})\end{array}$ & p-value & $\begin{array}{l}\text { Events } n \\
(\%)\end{array}$ & $\begin{array}{l}\text { Hazard ratio } \\
(95 \% \mathrm{Cl})\end{array}$ & $\mathrm{p}$-value \\
\hline \multicolumn{20}{|l|}{$\begin{array}{l}\text { Longer-acting opioid-only } \\
\text { agents" }\end{array}$} \\
\hline $\begin{array}{l}\leqslant 30 \mathrm{mg} \text { of morphine } \\
\text { equivalents per day }\end{array}$ & $\begin{array}{l}\text { New users } \\
\text { Controls }\end{array}$ & $\begin{array}{c}33(3.6) \\
1724(4.1)\end{array}$ & $\begin{array}{c}0.86 \text { (0.50-1.50) } \\
\text { Referent }\end{array}$ & 0.60 & $\begin{array}{l}19(2.0) \\
422(1.0)\end{array}$ & $\begin{array}{l}2.02(0.97-4.21) \\
\text { Referent }\end{array}$ & 0.06 & $\begin{array}{l}25(2.7) \\
588(1.4)\end{array}$ & $\begin{array}{l}1.93(1.05-3.54) \\
\text { Referent }\end{array}$ & 0.03 & $\begin{array}{c}<6^{+} \\
92.2)\end{array}$ & $\begin{array}{l}1.66(0.56-4.88) \\
\text { Referent }\end{array}$ & 0.36 & $\begin{array}{l}10(1.0) \\
71(0.2)\end{array}$ & $\begin{array}{l}6.46(2.94-14.21) \\
\text { Referent }\end{array}$ & $<0.0001$ & $\begin{array}{l}73(8.0) \\
510(1.2)\end{array}$ & $\begin{array}{l}6.89(5.03-9.44) \\
\text { Referent }\end{array}$ & $<0.0001$ \\
\hline $\begin{array}{l}>30 \mathrm{mg} \text { of morphine } \\
\text { equivalents per day }\end{array}$ & $\begin{array}{l}\text { New users } \\
\text { Controls }\end{array}$ & $\begin{array}{l}36(2.7) \\
1722(4.1)\end{array}$ & $\begin{array}{l}0.65(0.44-0.98) \\
\quad \text { Referent }\end{array}$ & 0.04 & $\begin{array}{l}15(1.2) \\
420(1.0)\end{array}$ & $\begin{array}{l}1.14(0.60-2.19) \\
\text { Referent }\end{array}$ & 0.69 & $\begin{array}{l}34(2.5) \\
586(1.4)\end{array}$ & $\begin{array}{l}1.82(1.04-3.17) \\
\text { Referent }\end{array}$ & 0.04 & $\begin{array}{c}<6^{+} \\
92(0.2)\end{array}$ & $\begin{array}{l}0.77(0.12-4.76) \\
\quad \text { Referent }\end{array}$ & 0.78 & $\begin{array}{l}10(0.8) \\
71(0.2)\end{array}$ & $\begin{array}{l}4.72(1.24-18.05) \\
\text { Referent }\end{array}$ & 0.02 & $\begin{array}{l}76(5.7) \\
508(1.2)\end{array}$ & $\begin{array}{l}4.86 \text { (3.47-6.79) } \\
\text { Referent }\end{array}$ & $<0.0001$ \\
\hline \multicolumn{20}{|l|}{$\begin{array}{l}\text { Shorter-acting opioid-only } \\
\text { agents" }\end{array}$} \\
\hline $\begin{array}{l}\leqslant 30 \mathrm{mg} \text { of morphine } \\
\text { equivalents per day }\end{array}$ & $\begin{array}{l}\text { New users } \\
\text { Controls }\end{array}$ & $\begin{array}{l}155(4.9) \\
1736(4.2)\end{array}$ & $\begin{array}{l}1.19(0.96-1.47) \\
\text { Referent }\end{array}$ & 0.12 & $\begin{array}{l}43(1.4) \\
429(1.0)\end{array}$ & $\begin{array}{l}1.33(0.94-1.90) \\
\text { Referent }\end{array}$ & 0.11 & $\begin{array}{l}78(2.5) \\
600(1.4)\end{array}$ & $\begin{array}{l}1.73(1.26-2.36) \\
\quad \text { Referent }\end{array}$ & 0.0006 & $\begin{array}{l}14(0.4) \\
94(0.2)\end{array}$ & $\begin{array}{l}1.92(0.88-4.19) \\
\text { Referent }\end{array}$ & 0.10 & $\begin{array}{l}39(1.3) \\
71(0.2)\end{array}$ & $\begin{array}{l}7.55(4.84-11.79) \\
\text { Referent }\end{array}$ & $<0.0001$ & $\begin{array}{l}195(6.2) \\
516(1.2)\end{array}$ & $\begin{array}{l}5.19(4.26-6.31) \\
\text { Referent }\end{array}$ & $<0.0001$ \\
\hline $\begin{array}{l}>30 \mathrm{mg} \text { of morphine } \\
\text { equivalents per day }\end{array}$ & $\begin{array}{l}\text { New users } \\
\text { Controls }\end{array}$ & $\begin{array}{l}423(6.0) \\
1745(4.2)\end{array}$ & $\begin{array}{l}1.45(1.28-1.64) \\
\text { Referent }\end{array}$ & $<0.0001$ & $\begin{array}{l}136(1.9) \\
430(1.0)\end{array}$ & $\begin{array}{c}1.87(1.47-2.39) \\
\text { Referent }\end{array}$ & $<0.0001$ & $\begin{array}{l}148(2.1) \\
597(1.4)\end{array}$ & $\begin{array}{l}1.47(1.19-1.81) \\
\text { Referent }\end{array}$ & 0.0004 & $\begin{array}{l}19(0.3) \\
95(0.2)\end{array}$ & $\begin{array}{l}\text { 1.17 (0.66-2.08) } \\
\text { Referent }\end{array}$ & 0.59 & $\begin{array}{l}38(0.5) \\
69(0.2)\end{array}$ & $\begin{array}{c}3.26(2.04-5.20) \\
\text { Referent }\end{array}$ & $<0.0001$ & $\begin{array}{l}274(3.9) \\
512(1.2)\end{array}$ & $\begin{array}{c}3.21(2.72-3.79) \\
\text { Referent }\end{array}$ & $<0.0001$ \\
\hline
\end{tabular}

COPD: chronic obstructive pulmonary disease; ICU: intensive care unit. \#: this analysis includes tablet/capsule and transdermal formulations. If individuals were on concomitant shorter-acting opioid agents, only the longer-acting opioid was considered when determining the daily opioid dose. ": this analysis includes only tablet/capsule formulations. Due the lack of complete dosing information for liquid opioid formulations in Ontario Drug Benefit programme, liquid opioid formulations were excluded from the dosing analysis. ${ }^{+}$: percentages are not presented, according to Institute of Clinical Evaluative Sciences reporting rules, because of small cell size.

\begin{tabular}{|c|c|c|c|c|c|c|c|c|c|c|c|c|c|c|c|c|c|c|c|}
\hline \multirow[t]{2}{*}{$\begin{array}{l}\text { COPD exacerbation } \\
\text { frequency }\end{array}$} & \multirow[t]{2}{*}{$\begin{array}{l}\text { Opioid use } \\
\text { status }\end{array}$} & \multicolumn{3}{|c|}{ Outpatient respiratory exacerbations } & \multicolumn{3}{|c|}{$\begin{array}{l}\text { Emergency room visits for COPD or } \\
\text { pneumonia }\end{array}$} & \multicolumn{3}{|c|}{ Hospitalisations for COPD or pneumonia } & \multicolumn{3}{|c|}{$\begin{array}{l}\text { ICU admissions during hospitalisations } \\
\text { for COPD or pneumonia }\end{array}$} & \multicolumn{3}{|c|}{ COPD or pneumonia-related mortality } & \multicolumn{3}{|c|}{ All-cause mortality } \\
\hline & & $\begin{array}{l}\text { Events n } \\
\qquad \%)\end{array}$ & $\begin{array}{l}\text { Hazard ratio } \\
(95 \% \mathrm{Cl})\end{array}$ & p-value & $\begin{array}{l}\text { Events } \mathrm{n} \\
(\%)\end{array}$ & $\begin{array}{l}\text { Hazard ratio } \\
(95 \% \mathrm{Cl})\end{array}$ & p-value & $\begin{array}{l}\text { Events n } \\
\qquad \%)\end{array}$ & $\begin{array}{c}\text { Hazard } \\
\text { ratio }(95 \% \mathrm{Cl})\end{array}$ & p-value & $\begin{array}{l}\text { Events n } \\
\quad(\%)\end{array}$ & $\begin{array}{c}\text { Hazard ratio } \\
(95 \% \mathrm{Cl})\end{array}$ & $p$-value & $\begin{array}{l}\text { Events } \mathrm{n} \\
(\%)\end{array}$ & $\begin{array}{c}\text { Hazard ratio } \\
(95 \% \mathrm{Cl})\end{array}$ & p-value & $\begin{array}{c}\text { Events } \mathrm{n} \\
(\%)\end{array}$ & $\begin{array}{c}\text { Hazard ratio } \\
(95 \% \mathrm{Cl})\end{array}$ & $\mathrm{p}$-value \\
\hline $\begin{array}{l}0 \text { exacerbations in the } \\
\text { year prior to index }\end{array}$ & $\begin{array}{c}\text { New users } \\
\text { Controls }\end{array}$ & $\begin{array}{l}1071(1.9) \\
578(2.3)\end{array}$ & $\begin{array}{l}0.85(0.76-0.95) \\
\text { Referent }\end{array}$ & 0.003 & $\begin{array}{l}325(0.6) \\
120(0.5)\end{array}$ & $\begin{array}{l}1.25(0.99-1.57) \\
\text { Referent }\end{array}$ & 0.06 & $\begin{array}{l}403(0.7) \\
160(0.6)\end{array}$ & $\begin{array}{l}1.16(0.95-1.40) \\
\text { Referent }\end{array}$ & 0.14 & $\begin{array}{l}53(0.1) \\
28(0.1)\end{array}$ & $\begin{array}{l}0.86 \text { (0.53-1.42) } \\
\text { Referent }\end{array}$ & 0.56 & $\begin{array}{l}77(0.1) \\
23(0.1)\end{array}$ & $\begin{array}{l}1.52(0.95-2.41) \\
\text { Referent }\end{array}$ & 0.08 & $\begin{array}{l}813(1.5) \\
224(0.9)\end{array}$ & $\begin{array}{l}1.67(1.39-2.02) \\
\text { Referent }\end{array}$ & $<0.0001$ \\
\hline $\begin{array}{l}\geqslant 1 \text { outpatient respiratory } \\
\text { exacerbations in the } \\
\text { year prior to index }\end{array}$ & $\begin{array}{l}\text { New users } \\
\text { Controls }\end{array}$ & $\begin{array}{l}1086(6.3) \\
516(6.9)\end{array}$ & $\begin{array}{l}0.90(0.80-1.01) \\
\text { Referent }\end{array}$ & 0.08 & $\begin{array}{l}135(0.8) \\
80(1.1)\end{array}$ & $\begin{array}{l}0.73(0.54-0.98) \\
\quad \text { Referent }\end{array}$ & 0.04 & $\begin{array}{l}212(1.2) \\
110(1.5)\end{array}$ & $\begin{array}{l}0.83(0.64-1.07) \\
\text { Referent }\end{array}$ & 0.16 & $\begin{array}{l}28(0.2) \\
15(0.2)\end{array}$ & $\begin{array}{l}0.77(0.41-1.45) \\
\quad \text { Referent }\end{array}$ & 0.43 & $\begin{array}{c}38(0.2) \\
<6^{\#}\end{array}$ & $\begin{array}{l}3.15(1.09-9.14) \\
\text { Referent }\end{array}$ & 0.03 & $\begin{aligned} 258(1.5) \\
62(0.8)\end{aligned}$ & $\begin{array}{l}1.79(1.35-2.39) \\
\quad \text { Referent }\end{array}$ & $<0.0001$ \\
\hline $\begin{array}{l}\geqslant 1 \text { exacerbations } \\
\text { requiring } \\
\text { presentation to } \\
\text { hospital in the year } \\
\text { prior to index }\end{array}$ & $\begin{array}{l}\text { New users } \\
\text { Controls }\end{array}$ & $\begin{array}{l}992(6.1) \\
600(6.7)\end{array}$ & $\begin{array}{l}0.91(0.81-1.02) \\
\text { Referent }\end{array}$ & 0.09 & $\begin{array}{l}500(3.1) \\
222(2.5)\end{array}$ & $\begin{array}{l}1.25(1.05-1.48) \\
\text { Referent }\end{array}$ & 0.01 & $\begin{array}{l}647(4.0) \\
314(3.5)\end{array}$ & $\begin{array}{l}1.14(0.98-1.33) \\
\text { Referent }\end{array}$ & 0.09 & $\begin{array}{l}99(0.6) \\
49(0.5)\end{array}$ & $\begin{array}{l}1.13(0.73-1.75) \\
\text { Referent }\end{array}$ & 0.59 & $\begin{array}{r}142(0.9) \\
31(0.3)\end{array}$ & $\begin{array}{l}2.55(1.71-3.80) \\
\text { Referent }\end{array}$ & $<0.0001$ & $\begin{array}{l}628(3.8) \\
186(2.1)\end{array}$ & $\begin{array}{l}1.89(1.59-2.24) \\
\text { Referent }\end{array}$ & $<0.0001$ \\
\hline
\end{tabular}

COPD: chronic obstructive pulmonary disease; ICU: intensive care unit. \#: percentage is not presented, according to Institute of Clinical Evaluative Sciences reporting rules, because of small cell size. 
In the overall community-dwelling cohort, only one adverse respiratory morbidity outcome was significantly increased among new opioid users (i.e., emergency room visits for COPD or pneumonia), outpatient respiratory exacerbations were significantly reduced and there were generally no increased adverse respiratory morbidity outcomes across differing COPD severity subgroups. These results may be in part explained by the significantly increased and competing risk of death that was observed among new opioid users. These findings may also be related to the fact that combination opioid/non-opioid drug formulations (which constitute close to $90 \%$ of new opioid use among older adults with COPD [1]) were included in the aforementioned analyses. Compared with opioid-only agents, combination opioid/non-opioid drug formulations generally contain less potent opioids (like codeine), lower opioid doses are generally achievable and some medications within the latter group contain caffeine, which may protect from opioid-related sedation (see online supplement 1 for a sensitivity analysis involving opioids with and without caffeine). In contrast, when one considers the generally more potent and non-caffeine-containing opioid-only agents among community-dwelling older adults, their use was found to be associated with several adverse respiratory morbidity outcomes, including outpatient respiratory exacerbations and emergency room visits and hospitalisations for COPD and pneumonia. No significant association was observed between new opioid-only use in the community and ICU admissions during hospitalisations for COPD or pneumonia, which is a clinically serious outcome. However, relatively small numbers experiencing this event may explain why this outcome was non-significant. While our results suggest that use of the generally less potent combination opioid/non-opioid drugs among community-dwelling older adults may be associated with less respiratory risk, frailer long-term care resident older adults display vulnerability for several serious adverse respiratory outcomes with use of these agents (online supplement 2).

New opioid use was also found to be associated with significantly increased respiratory-related and all-cause mortality in the overall community-dwelling cohort. The fact that associations with increased all-cause mortality persisted across all subgroup analyses, including in the subgroup of individuals with the least severe COPD, supports the robustness of this finding. A previous population-based study also found associations with increased all-cause mortality with use of high-dose opioid compounds, but respiratory-related mortality was not examined [20]. Our study clarifies that the increased mortality risk observed among new opioid users with COPD is in part respiratory related. Our finding of increased respiratory exacerbation risk (which is known to be associated with mortality [37]) among new opioid users may explain the higher frequency of COPD or pneumonia-related deaths. New opioid users also had more cancer-related deaths than controls (data not shown), suggesting that residual confounding might also partly influence the observed opioid-mortality association. However, associations with increased respiratory-related and all-cause mortality risk persisted, even when individuals with pre-existing cancer were excluded from analyses (see online supplement 1 for this sensitivity analysis).

Adverse respiratory outcomes were found to occur even among community-dwelling new users of shorter-acting opioid-only agents. This is consistent with previous studies involving benzodiazepines, in which adverse respiratory [29] and psychomotor [38] outcomes were observed with use of shorter-acting agents. Compared with longer-acting agents, shorter-acting opioid agents are known to be associated with poorer steady-state blood levels and greater drug level peaks, which may in turn explain the increased risk for adverse outcomes with this drug subclass [39]. In contrast to a previous study [20], adverse outcomes were observed to occur also among new users of lower dose opioids (i.e., $\leqslant 30 \mathrm{mg}$ morphine equivalents per day), potentially refuting that such lower opioid doses are indeed safe from respiratory perspective. Patient drug dosing discretion (and concomitant receipt of shorter-acting opioids among longer-acting opioid users) may explain why hazard ratios for adverse events were sometimes greater in the lower versus higher opioid dose categories. The fact that incident opioid use was associated with increased adverse respiratory outcomes in multiple sensitivity analyses supports that our findings are robust. The fact that risk factors for opioid receipt between new users and controls were well balanced using propensity score weighting, and that incident non-opioid medication dispensing was used to identify controls, also makes it less likely that our adverse respiratory outcome results are due to confounding.

There are several limitations. First, as with all observational studies, causation cannot be inferred and our estimates could still be contaminated by residual confounding. However, the consistency of adverse respiratory outcomes associated with new opioid use across multiple sensitivity analyses supports the robustness of our results. Second, clinical data potentially influencing COPD severity and outcomes (e.g., respiratory and anxiety symptoms and lung function measures) were not available. However, our analyses adjusted for other important indicators of COPD severity, including respiratory exacerbation history, which is known to be associated with severity of underlying airflow obstruction [40], risk of future exacerbations [32] and mortality [37]. Third, our COPD definition, while highly specific, had more modest sensitivity [25], which may make our results less generalisable to the entire older COPD population. Fourth, information on indication for opioid receipt was not available. Fifth, while we performed a drug 
dosing sensitivity analysis, our categorisation of opioid users into different daily drug use levels may not be fully accurate. This is because our drug database does not distinguish standing versus pro re nata medication prescriptions and because individuals are also often given some discretion regarding opioid drug frequency and dose used. These points are likely more relevant to shorter-acting opioids, as longer-acting opioids would likely be prescribed on a standing and stable dose basis. Finally, we did not evaluate for respiratory depression as an adverse outcome, because ICD code algorithms for respiratory depression have been found to have poor sensitivity [41].

In conclusion, among older adults with COPD, incident opioid use and in particular use of the generally more potent opioid-only formulations, regardless of dose, was associated with several significantly increased adverse respiratory outcomes and mortality. The fact that incident opioids are frequently initiated in older adults with COPD [1] makes these results particularly worrisome. Our findings suggest that a careful, individualised approach needs to be taken when administering opioids to older adults with COPD, given the potential for adverse respiratory outcomes.

\section{References}

1 Vozoris NT, Wang X, Fischer HD, et al. Incident opioid drug use among older adults with chronic obstructive pulmonary disease: a population-based cohort study. Br J Clin Pharmacol 2016; 81: 161-170.

2 Edmonds $\mathrm{P}$, Karlsen S, Khan S, et al. A comparison of the palliative care needs of patients dying from chronic respiratory diseases and lung cancer. Palliat Med 2001; 15: 287-295.

3 Elkington $\mathrm{H}$, White $\mathrm{P}$, Addington-Hall J, et al. The healthcare needs of chronic obstructive pulmonary disease patients in the last year of life. Palliat Med 2005; 19: 485-491.

4 Borge CR, Wahl AK, Moum T. Pain and quality of life with chronic obstructive pulmonary disease. Heart Lung 2011; 40: e90-e101.

$5 \quad$ Klink ME, Quan SF. Prevalence of reported sleep disturbances in a general adult population and their relationship to obstructive airways diseases. Chest 1987; 91: 540-546.

6 Cormick W, Olson LG, Hensley MJ, et al. Nocturnal hypoxaemia and quality of sleep in patients with chronic obstructive lung disease. Thorax 1986; 41: 846-854.

7 Elkington $\mathrm{H}$, White $\mathrm{P}$, Addington-Hall $\mathrm{J}$, et al. The healthcare needs of chronic obstructive pulmonary disease patients in the last year of life. Palliat Med 2005; 19: 485-491.

8 Gray PA, Rekling JC, Bocchiaro CM, et al. Modulation of respiratory frequency by peptidergic input to rhythmogenic neurons in the preBotzinger complex. Science 1999; 286: 1566-1568.

9 Montandon G, Qin W, Liu H, et al. PreBotzinger complex neurokinin-1 receptor-expressing neurons mediate opioid-induced respiratory depression. J Neurosci 2011; 31: 1292-1301.

10 Lalley PM. Opioid receptor agonist effects on medullary respiratory neurons in the cat: evidence for involvement in certain types of ventilatory disturbances. Am J Physiol Regul Integr Comp Physiol 2003; 285: R1287-R1304.

11 Weil JV, McCullough RE, Kline JS, et al. Diminished ventilatory response to hypoxia and hypercapnia after morphine in normal man. N Engl J Med 1975; 292: 1103-1106.

12 Adcock JJ, Schneider C, Smith TW. Effects of codeine, morphine and a novel opioid pentapeptide BW443C, on cough, nociception and ventilation in the unanaesthetized guinea-pig. Br J Pharmacol 1988; 93: 93-100.

13 Vallejo R, de Leon-Casasola O, Benyamin R. Opioid therapy and immunosuppression: a review. Am J Ther 2004; 11: 354-365.

14 Woodcock AA, Gross ER, Gellert A, et al. Effects of dihydrocodeine, alcohol, and caffeine on breathlessness and exercise tolerance in patients with chronic obstructive lung disease and normal blood gases. N Engl J Med 1981; 305: 1611-1616.

15 Johnson MA, Woodcock AA, Geddes DM. Dihydrocodeine for breathlessness in "pink puffers". Br Med J (Clin Res Ed) 1983; 286: 675-677.

16 Jennings AL, Davies AN, Higgins JP, et al. A systematic review of the use of opioids in the management of dyspnoea. Thorax 2002; 57: 939-944.

17 Abernethy AP, Currow DC, Frith P, et al. Randomised, double blind, placebo controlled crossover trial of sustained release morphine for the management of refractory dyspnoea. BMJ 2003; 327: 523-528.

18 Currow DC, McDonald C, Oaten S, et al. Once-daily opioids for chronic dyspnea: a dose increment and pharmacovigilance study. J Pain Symptom Manage 2011; 42: 388-399.

19 Rocker GM, Simpson AC, Joanne Young BHSc, et al. Opioid therapy for refractory dyspnea in patients with advanced chronic obstructive pulmonary disease: patients' experiences and outcomes. CMAJ Open 2013; 1: E27-E36.

20 Ekström M, Bornefalk-Hermansson A, Currow DC. Safety of benzodiazepines and opioids in very severe respiratory disease: national prospective study. BMJ 2014; 348: g445.

21 Lanken PN, Terry PB, Delisser HM, et al. An official American Thoracic Society clinical policy statement: palliative care for patients with respiratory diseases and critical illnesses. Am J Respir Crit Care Med 2008; 177: 912-927.

22 Mahler DA, Selecky PA, Harrod CG, et al. American College of Chest Physicians consensus statement on the management of dyspnea in patients with advanced lung or heart disease. Chest 2010; 137: 674-691.

23 Marciniuk D, Goodridge D, Hernandez P, et al. Managing dyspnea in patients with advanced chronic obstructive pulmonary disease: a Canadian Thoracic Society clinical practice guideline. Can Respir J 2011; 18: 69-78.

24 Global Initiative for Chronic Obstructive Lung Disease (GOLD). Global Strategy for the Diagnosis, Management, and Prevention of Chronic Obstructive Pulmonary Disease. 2014. www.goldcopd.com/uploads/users/files/GOLD_ Report_2014_Oct30.pdf Date last accessed: January, 2015.

25 Gershon AS, Wang C, Guan J, et al. Identifying individuals with physician diagnosed COPD in health administrative databases. J COPD 2009; 6: 388-394. 
Levy R, O’Brien BJ, Sellors C, et al. Coding accuracy of administrative drug claims in the Ontario Drug Benefit database. Can J Clin Pharmacol 2003; 10: 67-71.

27 Gershon AS, Wang C, Wilton AS, et al. Trends in chronic obstructive pulmonary disease prevalence, incidence, and mortality in Ontario, Canada, 1996 to 2007: a population-based study. Arch Intern Med 2010; 170: 560-565.

28 Rochon PA, Normand SL, Gomes T, et al. Antipsychotic therapy and short-term serious events in older adults with dementia. Arch Inter Med 2008; 168: 1090-1096.

29 Vozoris NT, Fischer HD, Wang X, et al. Benzodiazepine drug use and adverse respiratory outcomes among older adults with COPD. Eur Respir J 2014; 44: 332-340.

30 Austin PC. An Introduction to propensity score methods for reducing the effects of confounding in observational studies. Multivariate Behav Res 2011; 46: 399-424.

31 Austin PC, Stuart EA. Moving towards best practice when using inverse probability of treatment weighting (IPTW) using the propensity score to estimate causal treatment effects in observational studies. Stat Med 2015; 34: 3661-3679.

32 Hurst JR, Vestbo J, Anzueto A, et al. Susceptibility to exacerbation in chronic obstructive pulmonary disease. N Engl J Med 2010; 363: 1128-1138.

33 Donaldson GC, Hurst JR, Smith CJ, et al. Increased risk of myocardial infarction and stroke following exacerbation of COPD. Chest 2010; 137: 1091-1097.

34 Wen SW, Hernandez R, Naylor CD. Pitfalls in nonrandomized outcomes studies. The case of incidental appendectomy with open cholecystectomy. JAMA 1995; 274: 1687-1691.

35 Greenfield S. The state of outcomes research: are we on target? N Engl J Med 1989; 320: 1142-1143.

36 Austin PC. The performance of different propensity-score methods for estimating marginal hazard ratios Stat Med 2013; 32: 2837-2849.

37 Connors AF Jr, Dawson NV, Thomas C, et al. Outcomes following acute exacerbation of severe chronic obstructive lung disease. The SUPPORT investigators (Study to Understand Prognoses and Preferences for Outcomes and Risks of Treatments). Am J Respir Crit Care Med 1996; 154: 959-967.

38 Herings RM, Stricker BH, de Boer A, et al. Benzodiazepines and the risk of falling leading to femur fractures. Dosage more important than elimination half-life. Arch Intern Med 1995; 155: 1801-1807.

39 Argoff CE, Silvershein DI. A comparison of long- and short-acting opioids for the treatment of chronic noncancer pain: tailoring therapy to meet patient needs. Mayo Clin Proc 2009; 84: 602-612.

40 Donaldson GC, Seemungal TA, Bhowmik A, et al. Relationship between exacerbation frequency and lung function decline in chronic obstructive pulmonary disease. Thorax 2002; 57: 847-852.

41 Stein BD, Bautista A, Schumock GT, et al. The validity of International Classification of Diseases, Ninth Revision, Clinical Modification diagnosis codes for identifying patients hospitalized for COPD exacerbations. Chest 2012; 141: 87-93. 